\title{
Assessment of Drug Prescription Pattern in Two District Hospitals, Northwest Ethiopia
}

Muluken Wubetu ${ }^{1^{*}}$, Dagninet Derebe ${ }^{2}$, Tafere Mulaw ${ }^{1}$, Tenaw Yimer $^{3}$, Getachew Hailu

${ }^{1}$ Department of Pharmacy, College of Health Sciences, Debre Markos University, Ethiopia

${ }^{2}$ Department of Pharmacy, College of Medicine and Health Sciences, Bahir Dar University, Ethiopia

${ }^{3}$ Department of Public health, College of Health Sciences, Debre Markos University, Ethiopia

*Corresponding author: Muluken Wubetu, Department of Pharmacy, College of Health Sciences, Debre Markos University, Ethiopia, Tel: +251587711646; E-mail: mulukenwubetu1986@gmail.com

Received date: January 05, 2018; Accepted date: January 11, 2018; Published date: January 18, 2018

Copyright: (c) 2018 Wubetu M, et al. This is an open-access article distributed under the terms of the Creative Commons Attribution License, which permits unrestricted use, distribution, and reproduction in any medium, provided the original author and source are credited.

\begin{abstract}
Background: According to World Health Organization more than half of all medicines are prescribed and dispensed improperly, hence half of patients take them inappropriately. To reduce morbidity and mortality and their adverse outcomes, appropriate drug utilization has an enormous impact.
\end{abstract}

Methodology: This study is aimed to assess prescription patterns of drugs in Finote Selam and Motta District Hospital in outpatient pharmacy unit. The study was cross-sectional retrospective by selecting the outpatient pharmacy units of Finote Selam and Motta district hospitals. A total of 362 prescription papers were incorporated from March 01/2015 to February 29/2016. A structured standardized proforma, adopted from the world health organizations, was used to fill data by observing the prescriptions papers of patients. The data was entered into EPI INFO version 7.0, and then analyzed using World Health Organization drug use indicators.

Results: A total of 362 prescriptions were reviewed retrospectively from a total of those prescribed for 1-year period. The average number of drugs prescribed per encounter was 2.1 with a range between 1 and 5 . The percentage of encounters in which an antibiotic or injection was ordered was $58.8 \%$ and $3.6 \%$, respectively while the percentage of those drugs prescribed by generic name and from an essential drug list was $98.4 \%(n=742)$ and $81 \%$ $(n=611)$, respectively. Amoxicillin $(9.2 \%)$, ciprofloxacin $(6.2 \%)$, and doxycycline $(4.8 \%)$ were among the common antibiotics prescribed.

Conclusion: Our study showed that the prescription pattern for most antibiotics deviates from the one advised by the world health organization. There is also overuse of some antibiotics which needs urgent intervention. The study also revealed the occurrence of an increasing practice of prescribing drugs using generic names. The very interesting point that our study revealed is problems like poly pharmacy and prescribing from the essential drug list were not concerns in the studied hospitals. Baseline data gathered by this study can be used by researchers and policymakers to improve prescribing practice at these two Hospitals. Lastly, drug prescribing practices in these two hospitals are not far from the world health organization requirements.

Keywords: Essential drugs; Ethiopia; District hospital; Prescription pattern

\section{Abbreviations}

AIDS: Acquired Immunodeficiency Syndrome; EDL: Essential Drug List; HIV: Human Immune Deficiency Virus; WHO: World Health Organization.

\section{Introduction}

Medicines play an indispensible role in health care delivery and disease prevention of a given nation. The availability and affordability of good quality drugs along with their rational use is vital for effective health care. The contribution of drugs to treatment and prevention of diseases has risen more rapidly than most non pharmaceutical approaches to various ailments. However the patterns of drug prescription are often incorrect and the need for registration of these patterns is important to improve good prescribing habits [1].

Prescription writing is a science and an art as it reflects the instructions given by the prescribing physician to the client. A report of World Health Organization (WHO) shows that more than half of drugs are ordered, or dispensed inappropriately. This is a common practice in developing countries like Ethiopia. Such Inappropriate drug use is enormous in terms of both inadequate resources and the adverse clinical consequences of therapies that may have real risks [2-5].

There are three important core drug use indicators; namely, prescribing indicators, patient care indicators and facility indicators. For this study we have used prescribing indicators [6,7]. Antimicrobial resistance is the greatest challenge to the effective management of diseases that countries face globally. Resistance negatively affects financial and therapeutic results with effects ranging from the failure of patients to respond and the need for expensive drugs to the costs of increased morbidity and mortality [8-10]. 
The manner in which prescribers order many drugs reinforces patients beliefs that they need "a pill for every illness" and that being treated is impossible without taking medicines [11]. Data about drug usage patterns is not satisfactory. There is lack of data on prescription pattern studies. Keeping these facts in consideration, the present study was planned to assess the prescription pattern in the outpatient units of Mota and Finoteselam Hospitals.

\section{Methodology}

\section{Data collection and analysis}

First, the total prescriptions in the two hospitals were counted and a total of 75,332 were found in two hospitals 40,946 (in Finote Selam) and 34,386 (in Motta). The sample prescription was proportionally allocated to two hospitals 197 for Finote Selam and 165 for Motta hospital. Prescriptions containing drugs which are not readable and those containing medical supplies were also excluded from the study. Data collection tool was the one adopted from WHO.

Common parameter like prescriber's identification and drug related information were used to assess completeness of prescription. Legibility of prescriptions was also checked. The average numbers of drugs ordered per encounter were calculated by dividing the total number of drugs prescribed by the number of encounters sampled, it is computed to measure the degree of poly-pharmacy. Fixed dose combination products were considered as one drug. Proportion of medicines prescribed by generic name was determined to measure the tendency of prescribing by generic naming system. It was computed by dividing the number of drugs prescribed using generic nomenclature to total number of drugs.

Percentage of encounters in which antibiotics were given was calculated to measure the overall utility of antibiotics. It was computed by dividing the number of encounters with antibiotic use by the total number of encounters included, multiplied by 100 . By dividing the number of encounters with injection use by the total number of encounters surveyed, multiplied by 100 , we obtained fraction of encounters with an injection prescribed was calculated. To measure the degree to which practices conform to a national drug policy, percentage of drugs prescribed from an EDL was calculated.

\section{Results}

\section{Completeness of the prescription}

A total of 362 prescriptions from March 1/2015 to February 29/2016) were analyzed from the two hospitals. While assessing the completeness of the prescription, the demographic data of the patients was mentioned in all of the prescriptions in both hospitals but none of the prescription contains patient weight and address. The diagnosis of ailments was recorded in $64.66 \%$ and $54.67 \%$ of prescriptions in Motta and Finote Selam respectively. The route by which drugs were administered was mentioned in $80.64 \%$ and $69.67 \%$ of the prescriptions in Motta and Finote Selam respectively. Strength of the drugs was also expressed in $20.78 \%$ and $9.67 \%$ of prescriptions in Finote Selam and Motta respectively.

\section{Average number of drugs per encounter}

As shown in Figure 1, the average number of drugs per encounter was 2.37 at Motta and 1.84 at Finote Selam hospitals respectively; in this study we found the average number of prescription per encounter to be 2.1. About half of the prescriptions had less than three drugs per prescription.

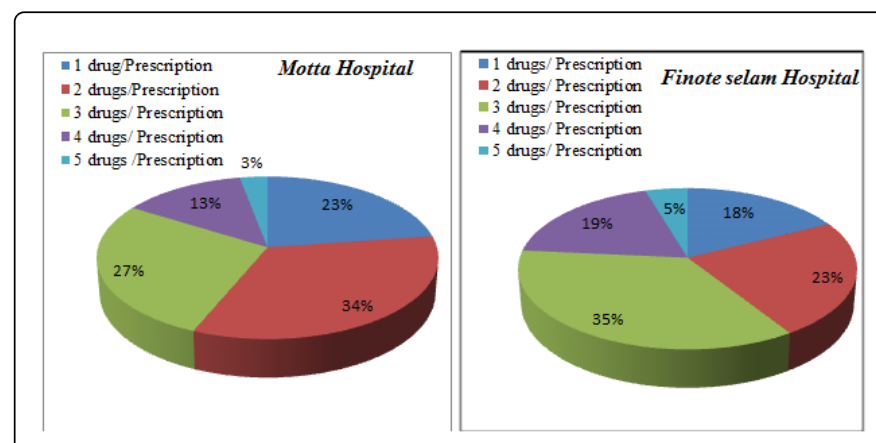

Figure 1: Distribution of drug prescription indicators in two hospitals.

\section{Percentage of drugs prescribed by generic name}

According to Table 1, the average percentage of generic drugs prescribed was comparable in both hospitals, this value is also nearly in agreement with the one recommended by WHO.

\begin{tabular}{|l|l|l|}
\hline Indicators & Motta & Finote selam \\
\hline Total number of prescriptions analyzed & 165 & 197 \\
\hline Total number of drugs prescribed & 391 & 363 \\
\hline Average number of drugs/prescription or encounter & 2.37 & 1.84 \\
\hline Percentage of drugs prescribed by generic name & 97.95 & 98.89 \\
\hline Percentage of drug prescribed from EDL/Formulary & 83.38 & 87.97 \\
\hline Percentage of prescriptions with antibiotics & 64.24 & 54.31 \\
\hline Percentage of Prescriptions having injection & 5.45 & 2.03 \\
\hline
\end{tabular}

Table 1: Analysis of prescriptions from the two district hospitals.

\section{Percentage of most commonly prescribed drugs}

Among the total drugs prescribed in different categories, $83 \%$ in Motta hospital and 79\% in Finote Selam hospital were prescribed from major nine groups of drugs. In both of the hospitals antimicrobials were the dominant class of drugs prescribed (37.34\% in Motta and $34.98 \%$ in Finote Selam) followed by Anti-inflammatory and anti-gout drugs.

\section{Percentage of encounters with an antibiotic}

The percentage of patients receiving antimicrobials was $64.24 \%$ in Motta and $54.31 \%$ Finote Selam district hospitals (Table 1). Antibiotic prescribing was much higher than the WHO standard. Of the antimicrobials amoxicillin was the commonly prescribed antibiotic (29.5\% in Motta and $19.7 \%$ in Finote Selam). 
Page 3 of 4

\section{Percentage of encounters with an injection prescribed and the routes of administration}

The average percentage of patients receiving one or more injections at the study hospitals was about $5.45 \%$ in Motta and $2.03 \%$ in Finote Selam hospital (Table 1). The most common route by which drugs were administered is oral route in both hospitals (Figure 2).

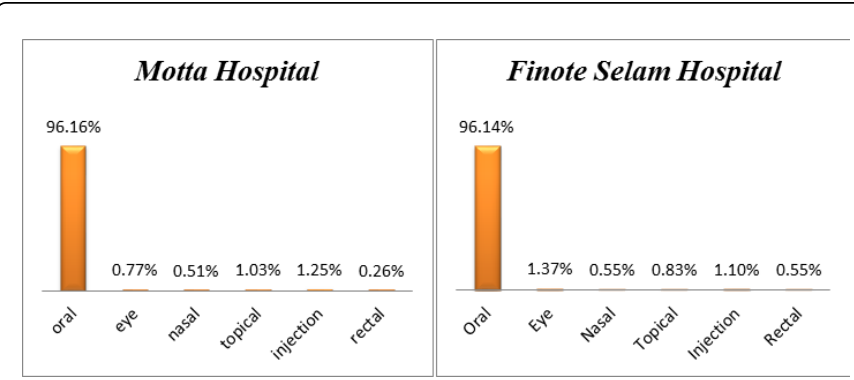

Figure 2: Route of drug administration.

\section{Percentage of drugs prescribed from EDL}

The average percentage of drugs prescribed from the EDL in the two hospitals was $83.38 \%$ and $87.97 \%$ in Motta and Finote Selam hospitals respectively (Table 1). In Motta, about $87 \%$ of essential dugs were available while in Finote Selam 90\% of them were presented.

\section{Discussion}

From this study, the average number of drugs ordered per prescription at Motta is 2.37, but at Finote Selam it is about 1.84, which is comparable with the standard [12]. However, our finding in Motta hospital is different from studies conducted at hospitals of Hawassa University, Jimma, Gondar [13-15]. According to a national baseline survey on drug use indicators, the average number of drugs ordered per prescription was 1.99, which is lower than Motta hospital, but higher than Finote Selam Hospital [16]. A study was conducted in 12 developing countries on drug use patterns and the result was high in Nigeria (3.8) and low in Zimbabwe (1.3)[17-19].

Concerning the percentage of drugs prescribed by generic name, the studied hospitals are almost in-line with the standard [12]. Majority of drugs in the two hospitals were from EDL. This may be due to the effectiveness of the procurement process and drug consumption in two Hospitals. This result is consistent with a study done at Hawassa, Zimbabwe and the one done nationally $[12,14,20]$ but different from those conducted at Jimma, Nigeria and Sudan [13,17-19].

The percentage encounters in which antimicrobials were ordered at Motta and Finote Selam were $64.24 \%$ and $54.31 \%$ respectively, which is very high compared to the standard [14]. This may be because of patients' expectation, beliefs on antibiotics and prescriber related aspects. This finding is also in agreement with results from other studies [17-19].

Interestingly, percentage of encounters in which injections were prescribed at Motta and Finote Selam were 5.45\% and 2.03\% respectively, which is much lower than the WHO requirement [14]. Professional and patient related intervention activities may be the contributing factors. However, our finding was in contrast with other related studies like in Uganda and Sudan [16-19].

\section{Conclusion}

Our study showed that the prescription pattern for most antibiotics deviates from the one advised by WHO. There is also overuse of some antibiotics which needs urgent intervention. The study also revealed the occurrence of a practice of prescribing drugs using generic names. The good point that our study revealed is problems like poly-pharmacy and prescribing from EDL were not concerns in the studied hospitals. Lastly, drug prescribing practices in these two hospitals are not far from the WHO requirements.

\section{Competing Interest}

The authors declare that they have no competing interests.

\section{Authors' Contributions}

MW, TM and DD contributed in the design, data collection and analysis of data. TY and GH were instrumental in supervising the data.

\section{References}

1. Bantie L (2014) Assessment of drug prescription practice using WHO prescribing indicators in Felege Hiwot referral hospital (FHRH) outpatient department, North Ethiopia. Int J Pharm 4: 89-94.

2. WHO (1987) The Rational use of drugs: report of the conference of experts, Nairobi, 25-29 November 1985: World Health Organization. p: 338.

3. Abula T, Desta Z, Yohannes AG (2004) Prescribing pattern of drugs in medical wards of three hospitals in northwest Ethiopia. Journal of Ethiopian Medical Practice 4: 8-13.

4. Aronson JK (2006) A prescription for better prescribing. British Journal of Clinical Pharmacology 61: 487-491.

5. Tamuno I, Fadare JO (2012) Drug prescription pattern in a Nigerian tertiary hospital. Tropical Journal of Pharmaceutical Research 11: 146-152.

6. WHO (2002) Promoting rational use of medicines: core components. Geneva. p: 6.

7. Kaiser BM. World Health Organization's Essential Medicines List: From Idea to Implementation. Global Health Education Consortium and collaborating partner's. p: 56.

8. Desse TA, Eshetie TC (2014) Assessment of drug use pattern using world health organization core drug use indicators at debre markos referral hospital. IJIPSR 2: 1270-1288.

9. Raza UA, Khursheed T, Irfan M, Abbas M, Irfan UM (2014) Prescription patterns of general practitioners in peshawar, pakistan. Pakistan Journal of Medical Sciences 30: 462-465.

10. Hangoma JM (2015) Antibiotic prescribing patterns among physicians at the University Teaching Hospital in Lusaka, Zambia.

11. Summoro TS, Gidebo KD, Kanche ZZ, Woticha EW (2015) Evaluation of trends of drug-prescribing patterns based on WHO prescribing indicators at outpatient departments of four hospitals in southern Ethiopia. Drug Design, Development and Therapy 9: 4551-4557.

12. WHO (1993) How to investigate drug use in health facilities: selected drug use indicators. Geneva:WHO/DAP/93.1: 199 p: 87.

13. Yenet W (2005) Baseline survey on drug prescribing indicators for outpatients in Jimma University Specialized Hospital, Southwest Ethiopia, ETHIOP Health Sci 15: 147-156.

14. Desalegn AA (2013) Assessment of drug use pattern using WHO prescribing indicators at Hawassa University teaching and referral hospital, south Ethiopia: a cross-sectional study. BMC Health Services Research 13: 170. 
Citation: Wubetu M, Derebe D, Mulaw T, Yimer T, Hailu G (2018) Assessment of Drug Prescription Pattern in Two District Hospitals, Northwest Ethiopia. J Health Educ Res Dev 6: 246. doi:10.4172/2380-5439.1000246

Page 4 of 4

15. Desta Z, Abula T, Gebre-Yohannes A, Worku A (2002) Drug prescribing patterns for outpatients in three hospitals in north-west Ethiopia. Ethiopian Journal of Health Development 16: 183-189.

16. Assessment of the Pharmaceutical Sector in Ethiopia. Addis Ababa; 2003.

17. (1992) Bimo: Report on Nigerian field test. INRUD News 3: 9-10.

18. In How to investigate drug use in health facilities. Geneva: WHO; 1993. 74(WHO/DAP/93.1).
19. Bannenberg WJ, Forshaw CJ, Fresle D, Salami AO, Wahab HA (1991) Evaluation of the Nile province essential drug project. WHO: Geneva; 1991. WHO/DAP/91.10.

20. (2017) Ministry of Health/Zimbabwe Essential Drugs Action Programme: Essential Drugs Survey. 